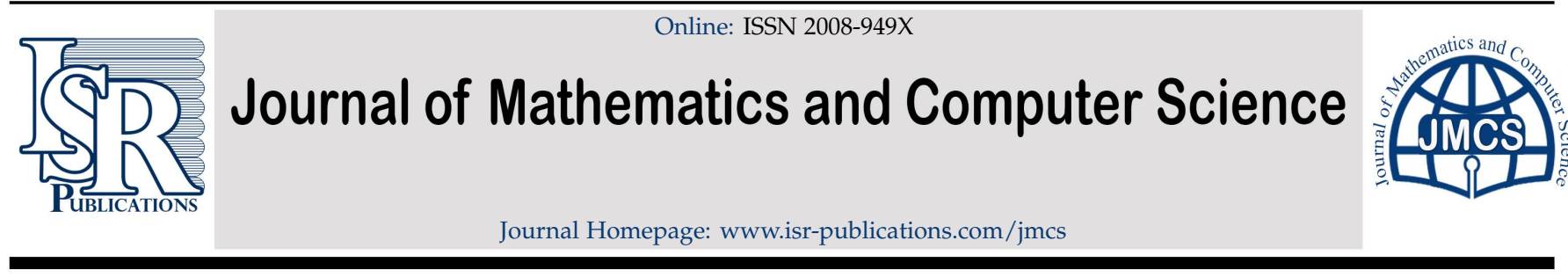

\title{
Coupled fixed points in complex partial metric spaces
}

\author{
M. Gunaseelan ${ }^{a}$, M. S. Khan ${ }^{b}$, Y. Mahendra Singh ${ }^{c}$, Kenan Tas ${ }^{d, *}$ \\ ${ }^{a}$ Department of Mathematics, Sri Sankara Arts and Science College(Autonomous), Enathur, Kanchipuram-631 561, India. \\ ${ }^{b}$ Department of Mathematics, College of Science, Sultan Qaboos University, P.O. Box 36, P. Code 123, Al Khod, Muscat, Oman. \\ ${ }^{c}$ Department of Humanities and Basic Sciences, Manipur Institute of Technology, A Constituent College of Manipur University, \\ Takyelpat-795004, Manipur, India. \\ ${ }^{d}$ Department of Mathematics, Cankaya University, Central Campus, 06790, Etimesgut, Ankara, Turkey.
}

\begin{abstract}
In this paper, we obtain coupled fixed point theorems in complex partial metric spaces under the different contractive conditions. Examples are provided to support our results.
\end{abstract}

Keywords: Coupled fixed point, complex valued metric space, complex partial metric space.

2020 MSC: 47H10, 54H25.

(C)2022 All rights reserved.

\section{Introduction}

In 1992, Matthews $([8,9])$ introduced the notion of partial metric space as a part of the study of denotational semantics of data flow programming languages in computer sciences and also extended Banach contraction principle in this space. Subsequently, many authors reported its topological properties and obtained a number of fixed point theorems (for more details and references, we refer to [6]). Azam et al. [3] introduced complex valued metric spaces and established the existence of common fixed point theorems of rational type contractions. Recently, Dhivya and Marudai [5] introduced complex partial metric space and established the existence of common fixed point theorems under the contraction condition of rational expression. Bhaskar and Lakshmikantham [4] introduced the concept of coupled fixed point. Círíc and Lakshmikantham [7] investigated some more coupled fixed point theorems in partially ordered sets. Aydi [2] proved some coupled fixed point results for mapping satisfying different contractive conditions on complete partial metric spaces. Also, Shatanawi et al. [13] extended the results of Aydi [2] in partial metric spaces. For more results obtained in this direction, we refer to ([1, 11-13]).

\footnotetext{
*Corresponding author

Email addresses: mathsguna@yahoo.com (M. Gunaseelan), mohammad@squ.edu.om (M. S. Khan), ymahenmit@rediffmail.com (Y. Mahendra Singh), kenan@cankaya.edu.tr (Kenan Tas)

doi: $10.22436 /$ jmcs.025.01.08
}

Received: 2020-04-12 Revised: 2021-03-05 Accepted: 2021-04-13 


\section{Preliminaries}

Let $\mathbb{C}$ be the set of complex numbers and $z_{1}, z_{2} \in \mathbb{C}$. Define a partial order $\preceq$ on $\mathbb{C}$ as follows: $z_{1} \preceq z_{2}$ if and only if $\operatorname{Re}\left(z_{1}\right) \leqslant \operatorname{Re}\left(z_{2}\right), \operatorname{Im}\left(z_{1}\right) \leqslant \operatorname{Im}\left(z_{2}\right)$. It follows that $z_{1} \preceq z_{2}$ if one of the following conditions is satisfied:

(C1) $\operatorname{Re}\left(z_{1}\right)=\operatorname{Re}\left(z_{2}\right), \operatorname{Im}\left(z_{1}\right)<\operatorname{Im}\left(z_{2}\right)$;

(C2) $\operatorname{Re}\left(z_{1}\right)<\operatorname{Re}\left(z_{2}\right), \operatorname{Im}\left(z_{1}\right)=\operatorname{Im}\left(z_{2}\right)$;

(C3) $\operatorname{Re}\left(z_{1}\right)<\operatorname{Re}\left(z_{2}\right), \operatorname{Im}\left(z_{1}\right)<\operatorname{Im}\left(z_{2}\right)$;

(C4) $\operatorname{Re}\left(z_{1}\right)=\operatorname{Re}\left(z_{2}\right), \operatorname{Im}\left(z_{1}\right)=\operatorname{Im}\left(z_{2}\right)$.

In particular, we write $z_{1} \precsim z_{2}$ if $z_{1} \neq z_{2}$ and one of (C1), (C2), and (C3) is satisfied and also we write $z_{1} \prec z_{2}$ if only (C3) is satisfied. Notice that

(a) $0 \preceq z_{1} \precsim z_{2}$, then $\left|z_{1}\right|<\left|z_{2}\right|$;

(b) $z_{1} \preceq z_{2}$ and $z_{2} \prec z_{3}$, then $z_{1} \prec z_{3}$;

(c) $0 \leqslant \mathrm{a} \leqslant \mathrm{b}$ and $z_{1} \preceq z_{2}$, then $\mathrm{a} z_{1} \preceq \mathrm{b} z_{2}$.

We collect some definitions with examples.

Definition 2.1 ([3]). Let $X$ be a non-empty set. A mapping $d_{c}: X \times X \rightarrow \mathbb{C}$ is called a complex valued metric on $X$ if it satisfies:

$\left(\mathrm{d}_{\mathfrak{c}} 1\right) 0 \preceq \mathrm{d}_{\mathfrak{c}}\left(z_{1}, z_{2}\right)$, for all $z_{1}, z_{2} \in X$ and $\mathrm{d}_{\mathfrak{c}}\left(z_{1}, z_{2}\right)=0 \Leftrightarrow z_{1}=z_{2}$;

$\left(\mathrm{d}_{\mathrm{c}} 2\right) \mathrm{d}_{\mathrm{c}}\left(z_{1}, z_{2}\right)=\mathrm{d}_{\mathrm{c}}\left(z_{2}, z_{1}\right)$, for all $z_{1}, z_{2} \in X$;

$\left(d_{\mathrm{c}} 3\right) \mathrm{d}_{\mathrm{c}}\left(z_{1}, z_{2}\right) \preceq \mathrm{d}_{\mathrm{c}}\left(z_{1}, z_{3}\right)+\mathrm{d}_{\mathrm{c}}\left(z_{3}, z_{2}\right)$, for all $z_{1}, z_{2}, z_{3} \in X$.

Then, $\left(X, d_{c}\right)$ is called a complex valued metric space.

Example 2.2. Let $X=\mathbb{C}$ be a set of complex number. Define $d_{c}: X \times X \rightarrow \mathbb{C}$ by

(i) $d_{c}\left(z_{1}, z_{2}\right)=\left|x_{1}-x_{2}\right|+i\left|y_{1}-y_{2}\right|$;

(ii) $\mathrm{d}_{\mathrm{c}}\left(z_{1}, z_{2}\right)=e^{i \theta}\left|z_{1}-z_{2}\right|$, where $z_{1}=x_{1}+i y_{1}, z_{2}=x_{2}+i y_{2}, 0 \leqslant \theta \leqslant \frac{\pi}{2}$.

Then, $\left(X, d_{c}\right)$ is a complex valued metric space.

Definition 2.3 ([8]). A partial metric on a non-empty set $X$ is a function $\sigma: X \times X \rightarrow \mathbb{R}^{+}$such that for all $p, q, r \in X:$

$\left(\sigma_{1}\right) \mathrm{p}=\mathrm{q} \Longleftrightarrow \sigma(\mathrm{p}, \mathrm{p})=\sigma(\mathrm{p}, \mathrm{q})=\sigma(\mathrm{p}, \mathrm{q}) ;$

$\left(\sigma_{2}\right) \sigma(p, p) \leqslant \sigma(p, q) ;$

$\left(\sigma_{3}\right) \sigma(p, q)=\sigma(q, p) ;$

$\left(\sigma_{4}\right) \sigma(p, q) \leqslant \sigma(p, q) \leqslant \sigma(p, r)+\sigma(r, q)-\sigma(r, r)$.

Then, the pair $(X, \sigma)$ is called a partial metric space. Note that if $\sigma(p, q)=0$, then from $(\sigma 1)$ and $(\sigma 2)$, $p=q$, however the converse may not be true.

Moreover, if $\sigma$ is a partial metric on $X$, then the function $d_{\sigma}: X \times X \rightarrow \mathbb{R}^{+}$defined by

$$
d_{\sigma}(p, q)=2 \sigma(p, q)-\sigma(p, p)-\sigma(q, q)
$$

is a metric on $X$.

Example 2.4 ([8,9]). Let $X=\mathbb{R}^{+}$and function $\sigma: X \times X \rightarrow \mathbb{R}^{+}$be defined by $\sigma(p, q)=\max \{p, q\}$, for all $p, q \in X$. Then, $(X, \sigma)$ is a partial metric space.

Example 2.5. Let $X=\{0,1,2\}$ and function $\sigma: X \times X \rightarrow \mathbb{R}^{+}$be defined by

$$
\sigma(0,0)=\sigma(1,1)=\sigma(2,2)=1 ; \quad \sigma(0,1)=\sigma(1,0)=\sigma(0,2)=\sigma(2,0)=2 ; \quad \sigma(1,2)=\sigma(2,1)=3 .
$$

Note that $\left(\sigma_{1}\right),\left(\sigma_{2}\right)$, and $\left(\sigma_{3}\right)$ are easy to verify. For $\left(\sigma_{4}\right)$, we have the following cases. 
Case (i): For $p=q, \sigma(p, q)=1$.

Case (ii): For $p=0, q=1$,

$$
2=\sigma(0,1) \leqslant \sigma(0,2)+\sigma(2,1)-\sigma(2,2)=4
$$

Case (iii): For $p=0, q=2$,

$$
2=\sigma(0,2) \leqslant \sigma(0,1)+\sigma(1,2)-\sigma(1,1)=4
$$

Case (iv): For $p=1, q=2$,

$$
3=\sigma(1,2) \leqslant \sigma(1,0)+\sigma(0,2)-\sigma(0,0)=3
$$

Then, $(X, \sigma)$ is a partial metric space.

Definition 2.6 ([5]). A complex partial metric on a non-empty set $Y$ is a function $\sigma_{c}: Y \times Y \rightarrow \mathbb{C}^{+}$such that for all $p, r, s \in Y$ :

$\left(\sigma_{\mathrm{c}} 1\right) 0 \preceq \sigma_{\mathrm{c}}(p, p) \preceq \sigma_{\mathcal{c}}(p, r)$ (small self-distances);

$\left(\sigma_{c} 2\right) \sigma_{c}(p, r)=\sigma_{c}(r, p)$ (symmetry);

$\left(\sigma_{c} 3\right) \sigma_{\mathfrak{c}}(p, p)=\sigma_{\mathcal{c}}(p, r)=\sigma_{\mathfrak{c}}(r, r) \Leftrightarrow p=r$ (equality);

$\left(\sigma_{\mathfrak{c}} 4\right) \sigma_{\mathfrak{c}}(p, r) \preceq \sigma_{\mathcal{c}}(p, s)+\sigma_{\mathcal{c}}(s, r)-\sigma_{\mathcal{c}}(s, s)$ (triangularity).

Then, $\left(\mathrm{Y}, \sigma_{\mathrm{c}}\right)$ is called a complex partial metric space and in short, we write CPMS.

For the complex partial metric $\sigma_{c}$ on $Y$, the function $\sigma_{c}^{t}: Y \times Y \rightarrow \mathbb{C}^{+}$given by $\sigma_{c}^{t}(p, r)=2 \sigma_{c}(p, r)-$ $\sigma_{\mathcal{c}}(p, p)-\sigma_{\mathcal{c}}(r, r)$ is a (usual) metric on $Y$. Each complex partial metric $\sigma_{\mathcal{c}}$ on $Y$ generates a topology $\tau_{\sigma_{c}}$ on $Y$ with the base family of open $\sigma_{\mathcal{c}}$-balls $\left\{B_{\sigma_{c}}(p, \epsilon): p \in Y, \epsilon \succ 0\right\}$, where $B_{\sigma_{c}}(p, \epsilon)=\left\{r \in Y: \sigma_{c}(p, r) \prec\right.$ $\left.\sigma_{\mathcal{c}}(p, p)+\epsilon\right\}$, for all $p \in Y$ and $0 \prec \epsilon \in \mathbb{C}^{+}$.

Note that a complex valued metric space is a CPMS. But a CPMS need not be a complex valued metric space. We discuss the following examples for complex partial metric spaces.

Example 2.7 ([5]). Let $Y=[0, \infty)$ endowed with complex partial metric $\sigma_{c}: Y \times Y \rightarrow \mathbb{C}^{+}$with $\sigma_{c}(p, q)=$ $\max \{p, q\}(1+i)$, for all $p, q \in Y$. Obviously $\left(Y, \sigma_{c}\right)$ is a complex partial metric space. Note that $\sigma_{\mathfrak{c}}(p, p)=$ $p+i p \neq 0$, for all $p \neq 0$. Therefore, $\left(Y, \sigma_{c}\right)$ is not a complex valued metric space.

Example 2.8. Let $Y=\mathbb{R}$ and function $\sigma_{\mathfrak{c}}: Y \times Y \rightarrow \mathbb{C}^{+}$be defined by $\sigma_{\mathfrak{c}}(p, q)=\{|p-q|+\tau\} e^{i \theta}$, for all $p, q \in Y$, where $\tau>0,0 \leqslant \theta \leqslant \frac{\pi}{2}$. It may be noted that $\sigma_{c}(p, p)=\tau e^{i \theta} \neq 0$, for all $p \in Y$. Obviously, one can verify that $\sigma_{\mathrm{c}}$ satisfies $\left(\sigma_{\mathrm{c}} 1\right),\left(\sigma_{\mathrm{c}} 2\right)$, and $\left(\sigma_{\mathrm{c}} 3\right)$ hold. For $\left(\sigma_{\mathrm{c}} 4\right)$, we obtain

$$
\begin{aligned}
\sigma_{\mathcal{c}}(p, q) & =\{|p-q|+\tau\} e^{i \theta} \\
& =\{|(p-r)+(r-q)|+\tau\} e^{i \theta} \\
& \preceq\{|p-r|+|r-q|+\tau\} e^{i \theta} \\
& =(\{|p-r|+\tau\}+\{|r-q|+\tau\}-\tau) e^{i \theta} \\
& =\sigma_{\mathcal{c}}(p, r)+\sigma_{\mathcal{c}}(r, q)-\sigma_{\mathcal{c}}(r, r) .
\end{aligned}
$$

Therefore, $\left(\mathrm{Y}, \sigma_{\mathrm{c}}\right)$ is a complex partial metric space.

Example 2.9. Let $X=\{0,1,2,3\}$ and $\sigma_{c}: X \times X \rightarrow \mathbb{C}^{+}$by

$$
\begin{aligned}
& \sigma_{\mathcal{c}}(0,0)=\sigma_{\mathcal{c}}(1,1)=\sigma_{\mathcal{c}}(2,2)=\sigma_{\mathcal{c}}(3,3)=e^{i \theta} ; \\
& \sigma_{\mathcal{c}}(0,1)=\sigma_{\mathcal{c}}(1,0)=\sigma_{\mathcal{c}}(0,2)=\sigma_{\mathcal{c}}(2,0)=\sigma_{\mathcal{c}}(0,3)=\sigma_{\mathcal{c}}(3,0)=2 e^{i \theta} ; \\
& \sigma_{\mathcal{c}}(1,2)=\sigma_{\mathcal{c}}(2,1)=\sigma_{\mathcal{c}}(1,3)=\sigma_{\mathcal{c}}(3,1)=\sigma_{\mathcal{c}}(2,3)=\sigma_{\mathcal{c}}(3,2)=3 e^{i \theta},
\end{aligned}
$$

where $0 \leqslant \theta \leqslant \frac{\pi}{2}$. One can verify that $\left(\sigma_{\mathrm{c}} 1\right),\left(\sigma_{\mathrm{c}} 2\right)$, and $\left(\sigma_{\mathrm{c}} 3\right)$. We verify $\left(\sigma_{\mathrm{c}} 4\right)$ with the following cases. 
Case (i): For $p=q \in X, \sigma_{c}(p, q)=e^{i \theta}$.

Case (ii): For $p=0, q=1, r \in\{2,3\}$, we obtain

$$
2 e^{i \theta}=\sigma_{\mathcal{c}}(0,1) \preceq \sigma_{\mathcal{c}}(0, r)+\sigma_{\mathcal{c}}(r, 1)-\sigma_{\mathcal{c}}(r, r)=4 e^{i \theta} .
$$

Case (iii): For $p=0, q=2, r \in\{1,3\}$, we obtain

$$
2 e^{i \theta}=\sigma_{\mathcal{c}}(0,2) \preceq \sigma_{\mathcal{c}}(0, r)+\sigma_{\mathcal{c}}(r, 2)-\sigma_{\mathcal{c}}(r, r)=4 e^{i \theta} .
$$

Case (iv): For $p=0, q=3, r \in\{1,2\}$, we obtain

$$
2 e^{i \theta}=\sigma_{\mathcal{c}}(0,3) \preceq \sigma_{\mathcal{c}}(0, r)+\sigma_{\mathcal{c}}(r, 3)-\sigma_{\mathcal{c}}(r, r)=4 e^{i \theta} .
$$

Case (v): For $p=1, q=2, r \in\{0,3\}$, we obtain

$$
3 e^{i \theta}=\sigma_{\mathcal{c}}(1,2) \preceq \sigma_{\mathcal{c}}(1, r)+\sigma_{\mathcal{c}}(r, 2)-\sigma_{\mathcal{c}}(r, r)=3 e^{i \theta}\left(\text { or }, 5 e^{i \theta}\right) .
$$

Case (vi): For $p=1, q=3, r \in\{0,2\}$, we obtain

$$
3 e^{i \theta}=\sigma_{\mathcal{c}}(1,3) \preceq \sigma_{\mathcal{c}}(1, r)+\sigma_{\mathcal{c}}(r, 3)-\sigma_{\mathcal{c}}(r, r)=3 e^{i \theta}\left(\text { or, } 5 e^{i \theta}\right) .
$$

Case (vii): For $p=2, q=3, r \in\{0,1\}$, we obtain

$$
3 e^{i \theta}=\sigma_{\mathcal{c}}(2,3) \preceq \sigma_{\mathcal{c}}(2, r)+\sigma_{\mathcal{c}}(r, 3)-\sigma_{\mathcal{c}}(r, r)=3 e^{i \theta}\left(\text { or }, 5 e^{i \theta}\right) .
$$

Therefore, $\left(X, \sigma_{c}\right)$ is a complex partial metric space.

Definition $2.10([5])$. Let $\left(Y, \sigma_{c}\right)$ be a complex partial metric space.

(1) A sequence $\left\{y_{n}\right\}$ in $\left(Y, \sigma_{c}\right)$ converges to $p \in Y$, if for every $0 \prec \in \in \mathbb{C}^{+}$there is $N \in \mathbb{N}$ such that for all $n>N$, we get $y_{n} \in B_{\sigma_{c}}(p, \epsilon)$. Then $p$ is said to be a limit of $\left\{y_{n}\right\}$, which is denoted by $\lim _{n \rightarrow \infty} y_{n}=p$ or, $y_{n} \rightarrow p$.

(2) A sequence $\left(y_{n}\right)$ in $\left(Y, \sigma_{c}\right)$ is called Cauchy if there is $a \in \mathbb{C}^{+}$such that for every $\epsilon>0$ there is $N \in \mathbb{N}$ such that for all $n, m \geqslant N,\left|\sigma_{c}\left(y_{n}, y_{m}\right)-a\right|<\epsilon$.

Definition $2.11([5])$. Let $\left(\mathrm{Y}, \sigma_{\mathrm{c}}\right)$ be a complex partial metric space.

(1) $\left(Y, \sigma_{c}\right)$ is said to be complete if every Cauchy sequence $\left\{y_{n}\right\}$ in $Y$ converges, with respect to $\tau_{\sigma_{c}}$, to a point $p \in Y$ such that $\sigma_{c}(p, p)=\lim _{n, m \rightarrow \infty} \sigma_{c}\left(y_{n}, y_{m}\right)$.

(2) A mapping $\mathrm{H}: \mathrm{Y} \rightarrow \mathrm{Y}$ is said to be continuous at $\mathrm{p}_{0} \in \mathrm{Y}$ if for every $\epsilon \succ 0$, there exists $\delta \succ 0$ such that $\mathrm{H}\left(\mathrm{B}_{\sigma_{\mathrm{c}}}\left(\mathrm{p}_{0}, \delta\right)\right) \subset \mathrm{B}_{\sigma_{\mathrm{c}}}\left(\mathrm{H}\left(\mathrm{p}_{0}\right), \epsilon\right)$.

Lemma $2.12([5])$. Let $\left(\mathrm{Y}, \sigma_{\mathrm{c}}\right)$ be a complex partial metric space. A sequence $\left\{\mathrm{y}_{\mathrm{n}}\right\}$ in $\left(\mathrm{Y}, \sigma_{\mathrm{c}}\right)$ converges to $\mathrm{p} \in \mathrm{Y}$ if and only if $\sigma_{c}(p, p)=\lim _{n \rightarrow \infty} \sigma_{c}\left(p, y_{n}\right)$.

Lemma $2.13([5])$. Let $\left(\mathrm{Y}, \sigma_{\mathrm{c}}\right)$ be a complex partial metric space. A sequence $\left\{\mathrm{y}_{\mathrm{n}}\right\}$ is Cauchy sequence in $\left(\mathrm{Y}, \sigma_{\mathrm{c}}\right)$, then $\left\{y_{n}\right\}$ is Cauchy in a metric space $\left(\mathrm{Y}, \sigma_{\mathrm{c}}^{\mathrm{t}}\right)$.

Lemma 2.14 ([10]). Let $\left(\mathrm{Y}, \sigma_{\mathrm{c}}\right)$ be a complex partial metric space.

(1) A sequence $\left\{\mathrm{y}_{\mathrm{n}}\right\}$ is Cauchy sequence in $\left(\mathrm{Y}, \sigma_{\mathrm{c}}\right)$ if and only if $\left\{\mathrm{y}_{\mathrm{n}}\right\}$ is Cauchy in a metric space $\left(\mathrm{Y}, \sigma_{\mathrm{c}}^{\mathrm{t}}\right)$.

(2) $\left(\mathrm{Y}, \sigma_{\mathrm{c}}\right)$ is complete if and only if $\left(\mathrm{Y}, \sigma_{\mathrm{c}}^{\mathrm{t}}\right)$ is complete. Moreover, $\lim _{\mathrm{n} \rightarrow \infty} \sigma_{\mathrm{c}}^{\mathrm{t}}\left(\mathrm{y}, \mathrm{y}_{\mathrm{n}}\right)=0 \Leftrightarrow \sigma_{\mathfrak{c}}(\mathrm{y}, \mathrm{y})=$ $\lim _{n \rightarrow \infty} \sigma_{c}\left(y, y_{n}\right)=\lim _{n, m \rightarrow \infty} \sigma_{c}\left(y_{n}, y_{m}\right)$.

Definition 2.15. Let $\left(Y, \sigma_{c}\right)$ be a complex partial metric space. Then an element $(p, r) \in Y \times Y$ is said to be a coupled fixed point of the mapping $F: Y \times Y \rightarrow Y$ if $F(p, r)=p$ and $F(r, p)=r$.

In this paper, we also extend the coupled fixed point results of Aydi [2] and Shatanawi et al. [13] in complex partial metric spaces. 


\section{Main results}

Theorem 3.1. Let $\left(\mathrm{Y}, \sigma_{\mathrm{c}}\right)$ be a complete CPMS. Suppose that the mapping $\psi: \mathrm{Y} \times \mathrm{Y} \rightarrow \mathrm{Y}$ satisfies

$$
\sigma_{\mathcal{c}}(\psi(p, q), \psi(r, s)) \preceq k \sigma_{\mathcal{c}}(p, r)+l \sigma_{\mathcal{c}}(q, s),
$$

for all $\mathrm{p}, \mathrm{q}, \mathrm{r}, \mathrm{s} \in \mathrm{Y}$, where $\mathrm{k}$ and $\mathrm{l}$ are non-negative constants with $\mathrm{k}+\mathrm{l}<1$. Then $\psi$ has a unique coupled fixed point.

Proof. Choose $p_{0}, q_{0} \in Y$ and set $p_{1}=\psi\left(p_{0}, q_{0}\right)$ and $q_{1}=\psi\left(q_{0}, p_{0}\right)$. Continuing in this process and setting $p_{n+1}=\psi\left(p_{n}, q_{n}\right)$ and $q_{n+1}=\psi\left(q_{n}, p_{n}\right)$, we obtain

$$
\sigma_{c}\left(p_{n}, p_{n+1}\right)=\sigma_{c}\left(\psi\left(p_{n-1}, q_{n-1}\right), \psi\left(p_{n}, q_{n}\right)\right) \preceq k \sigma_{c}\left(p_{n-1}, p_{n}\right)+l \sigma_{c}\left(q_{n-1}, q_{n}\right),
$$

which implies that

$$
\left|\sigma_{c}\left(p_{n}, p_{n+1}\right)\right| \leqslant k\left|\sigma_{c}\left(p_{n-1}, p_{n}\right)\right|+l\left|\sigma_{c}\left(q_{n-1}, q_{n}\right)\right| .
$$

Similarly, one can prove that

$$
\left|\sigma_{\mathcal{c}}\left(\mathbf{q}_{n}, \mathbf{q}_{n+1}\right)\right| \leqslant k\left|\sigma_{\mathcal{c}}\left(\mathbf{q}_{n-1}, \mathbf{q}_{n}\right)\right|+l\left|\sigma_{\mathcal{c}}\left(p_{n-1}, p_{n}\right)\right| .
$$

From (3.1) and (3.2), we get

$$
\begin{aligned}
\left|\sigma_{c}\left(p_{n}, p_{n+1}\right)\right|+\left|\sigma_{c}\left(q_{n}, q_{n+1}\right)\right| & \leqslant(k+l)\left(\left|\sigma_{c}\left(q_{n-1}, q_{n}\right)\right|+\left|\sigma_{c}\left(p_{n-1}, p_{n}\right)\right|\right) \\
& =\alpha\left(\left|\sigma_{c}\left(q_{n-1}, q_{n}\right)\right|+\left|\sigma_{c}\left(p_{n-1}, p_{n}\right)\right|\right),
\end{aligned}
$$

where $\alpha=k+l<1$. Also, we obtain

$$
\begin{aligned}
& \left|\sigma_{c}\left(p_{n+1}, p_{n+2}\right)\right| \leqslant k\left|\sigma_{c}\left(p_{n}, p_{n+1}\right)\right|+l\left|\sigma_{c}\left(q_{n}, q_{n+1}\right)\right| \\
& \left|\sigma_{c}\left(q_{n+1}, q_{n+2}\right)\right| \leqslant k\left|\sigma_{c}\left(q_{n}, q_{n+1}\right)\right|+l\left|\sigma_{c}\left(p_{n}, p_{n+1}\right)\right| .
\end{aligned}
$$

From (3.3) and (3.4), we obtain

$$
\begin{aligned}
\left|\sigma_{c}\left(p_{n+1}, p_{n+2}\right)\right|+\left|\sigma_{c}\left(q_{n+1}, q_{n+2}\right)\right| & \leqslant(k+l)\left(\left|\sigma_{c}\left(q_{n}, q_{n+1}\right)\right|+\left|\sigma_{c}\left(p_{n}, p_{n+1}\right)\right|\right) \\
& =\alpha\left(\left|\sigma_{c}\left(q_{n}, q_{n+1}\right)\right|+\left|\sigma_{c}\left(p_{n}, p_{n+1}\right)\right|\right) .
\end{aligned}
$$

Repeating this way, we obtain

$$
\begin{aligned}
\left|\sigma_{\mathcal{c}}\left(p_{n}, p_{n+1}\right)\right|+\left|\sigma_{c}\left(q_{n}, q_{n+1}\right)\right| & \leqslant \alpha\left(\left|\sigma_{c}\left(q_{n-1}, q_{n}\right)\right|+\left|\sigma_{c}\left(p_{n-1}, p_{n}\right)\right|\right) \\
& \leqslant \alpha^{2}\left(\left|\sigma_{c}\left(q_{n-2}, q_{n-1}\right)\right|+\left|\sigma_{c}\left(p_{n-2}, p_{n-1}\right)\right|\right) \\
& \vdots \\
& \leqslant \alpha^{n}\left(\left|\sigma_{c}\left(q_{0}, q_{1}\right)\right|+\left|\sigma_{c}\left(p_{0}, p_{1}\right)\right|\right) .
\end{aligned}
$$

Now, if $\left|\sigma_{c}\left(p_{n}, p_{n+1}\right)\right|+\left|\sigma_{c}\left(q_{n}, q_{n+1}\right)\right|=s_{n}$, then

$$
s_{n} \leqslant \alpha s_{n-1} \leqslant \cdots \leqslant \alpha^{n} s_{0} .
$$

If $s_{0}=0$, then $\left|\sigma_{c}\left(p_{0}, p_{1}\right)\right|+\left|\sigma_{c}\left(q_{0}, q_{1}\right)\right|=0$. Hence $p_{0}=p_{1}=\psi\left(p_{0}, q_{0}\right)$ and $q_{0}=q_{1}=\psi\left(q_{0}, p_{0}\right)$, which implies that $\left(p_{0}, q_{0}\right)$ is a coupled fixed point of $\psi$. Let $s_{0}>0$. For each $n \geqslant m$, we have

$$
\begin{aligned}
\sigma_{c}\left(p_{n}, p_{m}\right) \preceq & \sigma_{c}\left(p_{n}, p_{n-1}\right)+\sigma_{c}\left(p_{n-1}, p_{n-2}\right)-\sigma_{c}\left(p_{n-1}, p_{n-1}\right) \\
& +\sigma_{c}\left(p_{n-2}, p_{n-3}\right)+\sigma_{c}\left(p_{n-3}, p_{n-4}\right)-\sigma_{c}\left(p_{n-3}, p_{n-3}\right)
\end{aligned}
$$




$$
\begin{aligned}
& +\cdots+\sigma_{c}\left(p_{m+2}, p_{m+1}\right)+\sigma_{c}\left(p_{m+1}, p_{m}\right)-\sigma_{c}\left(p_{m+1}, p_{m+1}\right) \\
\preceq & \sigma_{c}\left(p_{n}, p_{n-1}\right)+\sigma_{c}\left(p_{n-1}, p_{n-2}\right)+\cdots+\sigma_{c}\left(p_{m+1}, p_{m}\right),
\end{aligned}
$$

which implies that

$$
\left|\sigma_{\mathcal{c}}\left(p_{n}, p_{m}\right)\right| \leqslant\left|\sigma_{\mathcal{c}}\left(p_{n}, p_{n-1}\right)\right|+\left|\sigma_{\mathcal{c}}\left(p_{n-1}, p_{n-2}\right)\right|+\cdots+\left|\sigma_{\mathcal{c}}\left(p_{m+1}, p_{m}\right)\right| .
$$

Similarly, one can prove that

$$
\left|\sigma_{\mathfrak{c}}\left(\mathrm{q}_{n}, \mathrm{q}_{\mathrm{m}}\right)\right| \leqslant\left|\sigma_{\mathfrak{c}}\left(\mathrm{q}_{\mathrm{n}}, \mathrm{q}_{\mathrm{n}-1}\right)\right|+\left|\sigma_{\mathfrak{c}}\left(\mathrm{q}_{\mathrm{n}-1}, \mathrm{q}_{\mathrm{n}-2}\right)\right|+\cdots+\left|\sigma_{\mathfrak{c}}\left(\mathrm{q}_{\mathrm{m}+1}, \mathrm{q}_{\mathrm{m}}\right)\right| .
$$

Thus,

$$
\begin{aligned}
\left|\sigma_{c}\left(p_{n}, p_{m}\right)\right|+\left|\sigma_{c}\left(q_{n}, q_{m}\right)\right| & \leqslant s_{n-1}+s_{n-2}+s_{n-3}+\cdots+s_{m} \\
& \leqslant\left(\alpha^{n-1}+\alpha^{n-2}+\cdots+\alpha^{m}\right) s_{0} \leqslant \frac{\alpha^{m}}{1-\alpha} s_{0} \rightarrow 0, \quad n \rightarrow \infty,
\end{aligned}
$$

which implies that $\left\{p_{n}\right\}$ and $\left\{q_{n}\right\}$ are Cauchy sequences in $\left(Y, \sigma_{c}\right)$. Since the complex partial metric space $\left(\mathrm{Y}, \sigma_{\mathrm{c}}\right)$ is complete, there exist $\mathrm{p}, \mathrm{q} \in \mathrm{Y}$ such that $\left\{\mathrm{p}_{\mathrm{n}}\right\} \rightarrow \mathrm{p}$ and $\mathrm{q}_{\mathrm{n}} \rightarrow \mathrm{q}$ as $\mathrm{n} \rightarrow \infty$ and $\sigma_{\mathrm{c}}(\mathrm{p}, \mathrm{p})=$ $\lim _{n \rightarrow \infty} \sigma_{\mathfrak{c}}\left(p, p_{n}\right)=\lim _{n, m \rightarrow \infty} \sigma_{\mathfrak{c}}\left(p_{n}, p_{m}\right)=0, \sigma_{\mathfrak{c}}(q, q)=\lim _{n \rightarrow \infty} \sigma_{\mathfrak{c}}\left(q, q_{n}\right)=\lim _{n, m \rightarrow \infty} \sigma_{\mathfrak{c}}\left(q_{n}, q_{m}\right)=$ 0 . We now show that $p=\psi(p, q)$. We suppose on the contrary that $p \neq \psi(p, q)$ and $q \neq \psi(q, p)$ so that $0 \prec \sigma_{\mathfrak{c}}(p, \psi(p, q))=l_{1}$ and $0 \prec \sigma_{\mathfrak{c}}(q, \psi(q, p))=l_{2}$, then

$$
\begin{aligned}
l_{1}=\sigma_{\mathfrak{c}}(p, \psi(p, q)) & \preceq \sigma_{\mathfrak{c}}\left(p, p_{n+1}\right)+\sigma_{\mathfrak{c}}\left(p_{n+1}, \psi(p, q)\right) \\
& =\sigma_{\mathfrak{c}}\left(p, p_{n+1}\right)+\sigma_{\mathcal{c}}\left(\psi\left(p_{n}, q_{n}\right), \psi(p, q)\right) \\
& \preceq \sigma_{c}\left(p, p_{n+1}\right)+k \sigma_{\mathfrak{c}}\left(p_{n}, p\right)+l \sigma_{\mathfrak{c}}\left(q_{n}, q\right),
\end{aligned}
$$

which implies that

$$
\left|l_{1}\right| \leqslant\left|\sigma_{\mathfrak{c}}\left(p, p_{n+1}\right)\right|+k\left|\sigma_{\mathfrak{c}}\left(p_{n}, p\right)\right|+l\left|\sigma_{\mathfrak{c}}\left(q_{n}, q\right)\right| .
$$

As $n \rightarrow \infty,\left|l_{1}\right| \leqslant 0$ which is a contradiction, therefore $\left|\sigma_{c}(p, \psi(p, q))\right|=0 \Longrightarrow p=\psi(p, q)$. Similarly we can prove that $q=\psi(q, p)$. Thus $(p, q)$ is a coupled fixed point of $\psi$. Now, if $(g, h)$ is another coupled fixed point of $\psi$, then

$$
\sigma_{\mathcal{c}}(p, g)=\sigma_{\mathcal{c}}(\psi(p, q), \psi(g, h)) \preceq k \sigma_{\mathcal{c}}(p, g)+l \sigma(q, h) .
$$

Thus,

$$
\sigma_{\mathcal{c}}(p, g) \preceq \frac{l}{1-k} \sigma_{\mathcal{c}}(q, h),
$$

which implies that

$$
\left|\sigma_{\mathfrak{c}}(p, g)\right| \leqslant \frac{l}{1-k}\left|\sigma_{\mathcal{c}}(q, h)\right|
$$

Similarly,

$$
\left|\sigma_{\mathrm{c}}(\mathrm{q}, \mathrm{h})\right| \leqslant \frac{\mathrm{l}}{1-\mathrm{k}}\left|\sigma_{\mathrm{c}}(\mathrm{p}, \mathrm{g})\right|
$$

From (3.5) and (3.6), we obtain

$$
\left|\sigma_{\mathfrak{c}}(p, g)\right|+\left|\sigma_{\mathcal{c}}(q, h)\right| \leqslant \frac{l}{1-k}\left[\left|\sigma_{\mathcal{c}}(p, g)\right|+\left|\sigma_{\mathcal{c}}(q, h)\right|\right], \quad\left(1-\frac{l}{1-k}\right)\left(\left|\sigma_{\mathcal{c}}(p, g)\right|+\left|\sigma_{\mathcal{c}}(q, h)\right|\right) \leqslant 0 .
$$

Since $k+l<1$, this implies that $\left|\sigma_{\mathcal{c}}(p, g)\right|+\left|\sigma_{\mathcal{c}}(q, h)\right| \leqslant 0$. Therefore $p=g$ and $q=h \Longrightarrow(p, q)=(g, h)$. Thus $\psi$ has a unique coupled fixed point. 
Corollary 3.2. Let $\left(\mathrm{Y}, \sigma_{\mathrm{c}}\right)$ be a complete CPMS. Suppose that the mapping $\psi: \mathrm{Y} \times \mathrm{Y} \rightarrow \mathrm{Y}$ satisfies

$$
\sigma_{c}(\psi(p, q), \psi(r, s)) \preceq \frac{k}{2}\left(\sigma_{c}(p, r)+\sigma_{c}(q, s)\right),
$$

for all $\mathrm{p}, \mathrm{q}, \mathrm{r}, \mathrm{s} \in \mathrm{Y}$, where $\mathrm{k}$ is a non-negative constant with $\mathrm{k}<1$. Then $\psi$ has a unique coupled fixed point.

Example 3.3. Let $Y=[0, \infty)$ endowed with the usual complex partial metric $\sigma_{c}: Y \times Y \rightarrow[0, \infty)$ defined by $\sigma_{c}(p, q)=\max \{p, q\}(1+i)$. The complex partial metric space $\left(Y, \sigma_{c}\right)$ is complete because $\left(Y, \sigma_{c}^{t}\right)$ is complete. Indeed, for any $p, q \in Y$,

$$
\sigma_{c}^{t}=2 \sigma_{c}(p, r)-\sigma_{c}(p, p)-\sigma_{c}(r, r)=2 \max \{p, q\}(1+i)-(p+i p)-(q+i q)=|p-q|+i|p-q| .
$$

Thus, $\left(\mathrm{Y}, \sigma_{\mathrm{c}}\right)$ is the Euclidean complex partial metric space which is complete. Consider the mapping $\psi: Y \times Y \rightarrow Y$ defined by $\psi(p, q)=\frac{p+q}{12}$. For any $p, q, g, h \in Y$, we have

$$
\begin{aligned}
\sigma_{c}(\psi(p, q), \psi(g, h)) & =\frac{1}{12} \max \{p+q, g+h\}(1+i) \\
& \preceq \frac{1}{12}[\max \{p, g\}+\max \{q, h\}](1+i)=\frac{1}{12}\left[\sigma_{c}(p, u)+\sigma_{c}(q, h)\right],
\end{aligned}
$$

which is the contractive condition (3.7) for $k=\frac{1}{6}$. Therefore, by Corollary 3.2, $\psi$ has a unique coupled fixed point, which is $(0,0)$. Note that if the mapping $\psi: Y \times Y \rightarrow Y$ is given by $\psi(p, q)=\frac{p+q}{2}$, then $\psi$ satisfies the contractive condition (3.7) for $k=1$, that is,

$$
\begin{aligned}
\sigma_{c}(\psi(p, q), \psi(g, h)) & =\frac{1}{2} \max \{p+q, g+h\}(1+i) \\
& \leqslant \frac{1}{2}[\max \{p, g\}+\max \{q, h\}](1+i) \\
& =\frac{1}{2}\left[\sigma_{c}(p, g)+\sigma_{c}(q, h)\right] .
\end{aligned}
$$

In this case, $(0,0)$ and $(1,1)$ are both coupled fixed points of $\psi$, and, hence, the coupled fixed point of $\psi$ is not unique. This shows that the condition $k<1$ in Corollary 3.2 and hence $k+l<1$ in Theorem 3.1 cannot be omitted in the statement of the aforesaid results.

Theorem 3.4. Let $\left(\mathrm{Y}, \sigma_{\mathrm{c}}\right)$ be a complete CPMS. Suppose that the mapping $\psi: \mathrm{Y} \times \mathrm{Y} \rightarrow \mathrm{Y}$ satisfies

$$
\sigma_{\mathcal{c}}(\psi(p, q), \psi(r, s)) \preceq k \sigma_{\mathcal{c}}(\psi(p, q), r)+l \sigma_{\mathcal{c}}(\psi(r, s), p),
$$

for all $\mathrm{p}, \mathrm{q}, \mathrm{r}, \mathrm{s} \in \mathrm{Y}$, where $\mathrm{k}$ and $\mathrm{l}$ are non-negative constants with $\mathrm{k}+2 \mathrm{l}<1$. Then $\psi$ has a unique coupled fixed point.

Proof. Choose $p_{0}, q_{0} \in Y$ and set $p_{1}=\psi\left(p_{0}, q_{0}\right)$ and $q_{1}=\psi\left(q_{0}, p_{0}\right)$. Continuing in this process and setting $p_{n+1}=\psi\left(p_{n}, q_{n}\right)$ and $q_{n+1}=\psi\left(q_{n}, p_{n}\right)$, we obtain

$$
\begin{aligned}
\sigma_{c}\left(p_{n}, p_{n+1}\right) & =\sigma_{c}\left(\psi\left(p_{n-1}, q_{n-1}\right), \psi\left(p_{n}, q_{n}\right)\right) \\
& \preceq k \sigma_{c}\left(\psi\left(p_{n-1}, q_{n-1}\right), p_{n}\right)+l \sigma_{c}\left(\psi\left(p_{n}, q_{n}\right), p_{n-1}\right) \\
& =k \sigma_{c}\left(p_{n}, p_{n}\right)+l \sigma_{c}\left(p_{n+1}, p_{n-1}\right) \\
& \preceq k \sigma_{c}\left(p_{n}, p_{n+1}\right)+l \sigma_{c}\left(p_{n+1}, p_{n-1}\right) \\
& \preceq k \sigma_{c}\left(p_{n}, p_{n+1}\right)+l\left(\sigma_{c}\left(p_{n+1}, p_{n}\right)+\sigma_{c}\left(p_{n}, p_{n-1}\right)-\sigma_{c}\left(p_{n}, p_{n}\right)\right) \\
& \preceq k \sigma_{c}\left(p_{n}, p_{n+1}\right)+l\left(\sigma_{c}\left(p_{n+1}, p_{n}\right)+\sigma_{c}\left(p_{n}, p_{n-1}\right)\right) \\
& \preceq \frac{l}{1-(k+l)} \sigma_{c}\left(p_{n}, p_{n-1}\right),
\end{aligned}
$$


which implies that

$$
\left|\sigma_{c}\left(p_{n}, p_{n+1}\right)\right| \leqslant \frac{l}{1-(k+l)}\left|\sigma_{c}\left(p_{n}, p_{n-1}\right)\right| .
$$

Similarly, one can prove that

$$
\left|\sigma_{\mathfrak{c}}\left(\mathrm{q}_{\mathrm{n}}, \mathrm{q}_{\mathrm{n}+1}\right)\right| \leqslant \frac{\mathrm{l}}{1-(\mathrm{k}+\mathrm{l})}\left|\sigma_{\mathrm{c}}\left(\mathrm{q}_{\mathrm{n}}, \mathrm{q}_{\mathrm{n}-1}\right)\right| .
$$

From (3.8) and (3.9), we obtain

$$
\begin{aligned}
\left|\sigma_{c}\left(p_{n}, p_{n+1}\right)\right|+\left|\sigma_{c}\left(q_{n}, q_{n+1}\right)\right| & \leqslant \frac{l}{1-(k+l)}\left(\left|\sigma_{c}\left(p_{n}, p_{n-1}\right)\right|+\left|\sigma_{c}\left(q_{n}, q_{n-1}\right)\right|\right) \\
& =\alpha\left(\left|\sigma_{c}\left(p_{n}, p_{n-1}\right)\right|+\left|\sigma_{c}\left(q_{n}, q_{n-1}\right)\right|\right),
\end{aligned}
$$

where $\alpha=\frac{l}{1-(k+l)}<1$. Also,

$$
\begin{aligned}
& \left|\sigma_{c}\left(p_{n+1}, p_{n+2}\right)\right| \leqslant \frac{l}{1-(k+l)}\left|\sigma_{c}\left(p_{n}, p_{n-1}\right)\right|, \\
& \left|\sigma_{c}\left(q_{n+1}, q_{n+2}\right)\right| \leqslant \frac{l}{1-(k+l)}\left|\sigma_{c}\left(q_{n}, q_{n-1}\right)\right| .
\end{aligned}
$$

From (3.10) and (3.11), we obtain

$$
\begin{aligned}
\left|\sigma_{c}\left(p_{n+1}, p_{n+2}\right)\right|+\left|\sigma_{c}\left(q_{n+1}, q_{n+2}\right)\right| & \leqslant \frac{l}{1-(k+l)}\left(\left|\sigma_{c}\left(p_{n}, p_{n-1}\right)\right|+\left|\sigma_{c}\left(q_{n}, q_{n-1}\right)\right|\right) \\
& =\alpha\left(\left|\sigma_{c}\left(p_{n}, p_{n-1}\right)\right|+\left|\sigma_{c}\left(q_{n}, q_{n-1}\right)\right|\right) .
\end{aligned}
$$

Repeating this way, we obtain

$$
\begin{aligned}
\left|\sigma_{\mathfrak{c}}\left(p_{n}, p_{n+1}\right)\right|+\left|\sigma_{\mathcal{c}}\left(q_{n}, q_{n+1}\right)\right| & \leqslant \alpha\left(\left|\sigma_{\mathcal{c}}\left(p_{n}, p_{n-1}\right)\right|+\left|\sigma_{\mathfrak{c}}\left(q_{n}, q_{n-1}\right)\right|\right) \\
& \leqslant \alpha^{2}\left(\left|\sigma_{c}\left(q_{n-2}, q_{n-1}\right)\right|+\left|\sigma_{c}\left(p_{n-2}, p_{n-1}\right)\right|\right) \\
& \vdots \\
& \leqslant \alpha^{n}\left(\left|\sigma_{\mathfrak{c}}\left(q_{0}, q_{1}\right)\right|+\left|\sigma_{c}\left(p_{0}, p_{1}\right)\right|\right) .
\end{aligned}
$$

Now, if $\left|\sigma_{\mathcal{c}}\left(p_{n}, p_{n+1}\right)\right|+\left|\sigma_{\mathcal{c}}\left(q_{n}, q_{n+1}\right)\right|=s_{n}$, then

$$
s_{n} \leqslant \alpha s_{n-1} \leqslant \cdots \leqslant \alpha^{n} s_{0} .
$$

If $s_{0}=0$ then $\left|\sigma_{\mathfrak{c}}\left(p_{0}, q_{1}\right)\right|+\left|\sigma_{\mathfrak{c}}\left(q_{0}, q_{1}\right)\right|=0$. Hence $p_{0}=p_{1}=\psi\left(p_{0}, q_{0}\right)$ and $q_{0}=q_{1}=\psi\left(q_{0}, p_{0}\right)$, which implies that $\left(p_{0}, q_{0}\right)$ is a coupled fixed point of $\psi$. Let $s_{0}>0$. For each $n \geqslant m$, we have

$$
\begin{aligned}
\sigma_{c}\left(p_{n}, p_{m}\right) \preceq & \sigma_{c}\left(p_{n}, p_{n-1}\right)+\sigma_{c}\left(p_{n-1}, p_{n-2}\right)-\sigma_{c}\left(p_{n-1}, p_{n-1}\right) \\
& +\sigma_{\mathcal{c}}\left(p_{n-2}, p_{n-3}\right)+\sigma_{c}\left(p_{n-3}, p_{n-4}\right)-\sigma_{c}\left(p_{n-3}, p_{n-3}\right) \\
& +\cdots+\sigma_{\mathcal{c}}\left(p_{m+2}, p_{m+1}\right)+\sigma_{\mathfrak{c}}\left(p_{m+1}, p_{m}\right)-\sigma_{\mathcal{c}}\left(p_{m+1}, p_{m+1}\right) \\
\preceq & \sigma_{\mathcal{c}}\left(p_{n}, p_{n-1}\right)+\sigma_{c}\left(p_{n-1}, p_{n-2}\right)+\cdots+\sigma_{\mathcal{c}}\left(p_{m+1}, p_{m}\right),
\end{aligned}
$$

which implies that

$$
\left|\sigma_{c}\left(p_{n}, p_{m}\right)\right| \leqslant\left|\sigma_{c}\left(p_{n}, p_{n-1}\right)\right|+\left|\sigma_{c}\left(p_{n-1}, p_{n-2}\right)\right|+\cdots+\left|\sigma_{c}\left(p_{m+1}, p_{m}\right)\right|
$$


Similarly, one can prove that

$$
\left|\sigma_{c}\left(q_{n}, q_{m}\right)\right| \leqslant\left|\sigma_{c}\left(q_{n}, q_{n-1}\right)\right|+\left|\sigma_{c}\left(q_{n-1}, q_{n-2}\right)\right|+\cdots+\left|\sigma_{c}\left(q_{m+1}, q_{m}\right)\right| .
$$

Thus,

$$
\begin{aligned}
\left|\sigma_{c}\left(p_{n}, p_{m}\right)\right|+\left|\sigma_{c}\left(q_{n}, q_{m}\right)\right| & \leqslant s_{n-1}+s_{n-2}+s_{n-3}+\cdots+s_{m} \\
& \leqslant\left(\alpha^{n-1}+\alpha^{n-2}+\cdots+\alpha^{m}\right) s_{0} \leqslant \frac{\alpha^{m}}{1-\alpha} s_{0} \rightarrow 0, \quad n \rightarrow \infty,
\end{aligned}
$$

which implies that $\left\{p_{n}\right\}$ and $\left\{q_{n}\right\}$ are Cauchy sequences in $\left(Y, \sigma_{c}\right)$. Since the complex partial metric space $\left(\mathrm{Y}, \sigma_{\mathrm{c}}\right)$ is complete, there exist $\mathrm{p}, \mathrm{q} \in \mathrm{Y}$ such that $\left\{\mathrm{p}_{\mathrm{n}}\right\} \rightarrow \mathrm{p}$ and $\mathrm{q}_{\mathrm{n}} \rightarrow \mathrm{q}$ as $\mathrm{n} \rightarrow \infty$ and $\sigma_{\mathrm{c}}(\mathrm{p}, \mathrm{p})=$ $\lim _{n \rightarrow \infty} \sigma_{c}\left(p, p_{n}\right)=\lim _{n, m \rightarrow \infty} \sigma_{c}\left(p_{n}, p_{m}\right)=0, \sigma_{c}(q, q)=\lim _{n \rightarrow \infty} \sigma_{c}\left(q, q_{n}\right)=\lim _{n, m \rightarrow \infty} \sigma_{c}\left(q_{n}, q_{m}\right)=$ 0 . We now show that $p=\psi(p, q)$. We suppose on the contrary that $p \neq \psi(p, q)$ and $q \neq \psi(q, p)$ so that $0 \prec \sigma_{\mathcal{c}}(p, \psi(p, q))=l_{1}$ and $0 \prec \sigma_{\mathcal{c}}(q, \psi(q, p))=l_{2}$, then

$$
\begin{aligned}
l_{1}=\sigma_{c}(p, \psi(p, q)) & \preceq \sigma_{c}\left(p, p_{n+1}\right)+\sigma_{\mathcal{c}}\left(p_{n+1}, \psi(p, q)\right) \\
& =\sigma_{c}\left(p, p_{n+1}\right)+\sigma_{c}\left(\psi\left(p_{n}, q_{n}\right), \psi(p, q)\right) \\
& \preceq \sigma_{c}\left(p, p_{n+1}\right)+k \sigma_{c}\left(\psi\left(p_{n}, q_{n}\right), p\right)+l \sigma_{c}\left(\psi(p, q), p_{n}\right) \\
& =\sigma_{c}\left(p, p_{n+1}\right)+k \sigma_{c}\left(p_{n+1}, p\right)+l \sigma_{c}\left(\psi(p, q), p_{n}\right),
\end{aligned}
$$

which implies that

$$
\left|l_{1}\right| \leqslant\left|\sigma_{c}\left(p, p_{n+1}\right)\right|+k\left|\sigma_{c}\left(p_{n}, p\right)\right|+l\left|\sigma_{c}\left(\psi(p, q), p_{n}\right)\right| .
$$

As $n \rightarrow \infty,\left|l_{1}\right| \leqslant 0$ which is a contradiction, therefore $\left|\sigma_{c}(p, \psi(p, q))\right|=0 \Longrightarrow p=\psi(p, q)$. Similarly, we can prove that $q=\psi(q, p)$. Thus $(p, q)$ is a coupled fixed point of $\psi$. Now, if $(g, h)$ is another coupled fixed point of $\psi$, then we obtain

$$
\sigma_{c}(p, g)=\sigma_{c}(\psi(p, q), \psi(g, h)) \preceq k \sigma_{c}(\psi(p, q), g)+l \sigma(\psi(g, h), p) .
$$

Thus,

$$
(1-(k+l)) \sigma_{c}(p, g) \preceq 0,
$$

which implies that

$$
(1-(k+l))\left|\sigma_{c}(p, g)\right| \leqslant 0 .
$$

Similarly,

$$
(1-(k+l))\left|\sigma_{c}(q, h)\right| \leqslant 0 .
$$

From (3.12) and (3.13), we obtain

$$
(1-(k+l))\left(\left|\sigma_{c}(p, g)\right|+\left|\sigma_{c}(q, h)\right|\right) \leqslant 0 .
$$

Since $k+l<1$, therefore $\left|\sigma_{\mathcal{c}}(p, g)\right|+\left|\sigma_{\mathcal{c}}(q, h)\right|=0$ and hence, $p=g$ and $q=h \Longrightarrow(p, q)=(g, h)$. Thus, $\psi$ has a unique coupled fixed point.

From Theorem 3.4 with $k=l$, we get the following corollary.

Corollary 3.5. Let $\left(\mathrm{Y}, \sigma_{\mathrm{c}}\right)$ be a complete CPMS. Suppose that the mapping $\psi: \mathrm{Y} \times \mathrm{Y} \rightarrow \mathrm{Y}$ satisfies

$$
\sigma_{\mathcal{c}}(\psi(p, q), \psi(r, s)) \preceq k\left(\sigma_{\mathcal{c}}(\psi(p, q), r)+\sigma_{\mathcal{c}}(\psi(r, s), p)\right),
$$

for all $\mathrm{p}, \mathrm{q}, \mathrm{r}, \mathrm{s} \in \mathrm{Y}$, where $\mathrm{k}$ is a non-negative constant with $\mathrm{k}<\frac{1}{3}$. Then $\psi$ has a unique coupled fixed point. 
Theorem 3.6. Let $\left(\mathrm{Y}, \sigma_{\mathrm{c}}\right)$ be a complete CPMS. Suppose that the mapping $\psi: \mathrm{Y} \times \mathrm{Y} \rightarrow \mathrm{Y}$ satisfies

$$
\sigma_{\mathcal{c}}(\psi(p, q), \psi(r, s)) \preceq r \max \left\{\sigma_{\mathfrak{c}}(p, r), \sigma_{\mathfrak{c}}(q, s), \sigma_{\mathcal{c}}(\psi(p, q), p), \sigma_{\mathfrak{c}}(\psi(r, s), r)\right\},
$$

for all $\mathrm{p}, \mathrm{q}, \mathrm{r}, \mathrm{s} \in \mathrm{Y}$, where $\mathrm{r} \in[0,1)$. Then $\psi$ has a unique coupled fixed point.

Proof. Choose $p_{0}, q_{0} \in Y$ and set $p_{1}=\psi\left(p_{0}, q_{0}\right)$ and $q_{1}=\psi\left(q_{0}, p_{0}\right)$. Continuing this process, set $p_{n+1}=$ $\psi\left(p_{n}, q_{n}\right)$ and $q_{n+1}=\psi\left(q_{n}, p_{n}\right)$. Then,

$$
\begin{aligned}
\sigma_{c}\left(p_{n+1}, p_{n+2}\right) & =\sigma_{c}\left(\psi\left(p_{n}, q_{n}\right), \psi\left(p_{n+1}, q_{n+1}\right)\right) \\
& \preceq r \max \left(\sigma_{c}\left(p_{n}, p_{n+1}\right), \sigma_{c}\left(q_{n}, q_{n+1}\right), \sigma_{c}\left(\psi\left(p_{n}, q_{n}\right), p_{n}\right), \sigma_{c}\left(\psi\left(p_{n+1}, q_{n+1}\right), p_{n+1}\right)\right. \\
& =r \max \left(\sigma_{c}\left(p_{n}, p_{n+1}\right), \sigma_{c}\left(q_{n}, q_{n+1}\right), \sigma_{c}\left(p_{n+1}, p_{n}\right), \sigma_{c}\left(p_{n+2}, p_{n+1}\right)\right) \\
& \preceq r \operatorname{rax}\left(\sigma_{c}\left(p_{n}, p_{n+1}\right), \sigma_{c}\left(q_{n}, q_{n+1}\right)\right),
\end{aligned}
$$

which implies that

$$
\left|\sigma_{c}\left(p_{n+1}, p_{n+2}\right)\right| \leqslant \max \left\{\left|\sigma_{c}\left(p_{n}, p_{n+1}\right)\right|,\left|\sigma_{c}\left(q_{n}, q_{n+1}\right)\right|\right\}
$$

Similarly, one can prove that

$$
\left|\sigma_{c}\left(q_{n+1}, q_{n+2}\right)\right| \leqslant r \max \left\{\left|\sigma_{\mathcal{c}}\left(q_{n}, q_{n+1}\right)\right|,\left|\sigma_{c}\left(p_{n}, p_{n+1}\right)\right|\right\} .
$$

From (3.14) and (3.15), we get

$$
\max \left\{\left|\sigma_{c}\left(p_{n+1}, p_{n+2}\right)\right|,\left|\sigma_{c}\left(q_{n+1}, q_{n+2}\right)\right|\right\} \leqslant \max \left\{\left|\sigma_{c}\left(q_{n}, q_{n+1}\right)\right|,\left|\sigma_{c}\left(p_{n}, p_{n+1}\right)\right|\right\} .
$$

Continuing this process, we obtain

$$
\begin{aligned}
\max \left\{\left|\sigma_{c}\left(p_{n}, p_{n+1}\right)\right|,\left|\sigma_{c}\left(q_{n}, q_{n+1}\right)\right|\right\} & \leqslant r \max \left\{\left|\sigma_{c}\left(q_{n-1}, q_{n}\right)\right|,\left|\sigma_{c}\left(p_{n-1}, p_{n}\right)\right|\right\} \\
& \leqslant r^{2} \max \left\{\left|\sigma_{c}\left(q_{n-2}, q_{n-1}\right)\right|,\left|\sigma_{c}\left(p_{n-2}, p_{n-1}\right)\right|\right\} \\
& \leqslant r^{n} \max \left\{\left|\sigma_{c}\left(q_{0}, q_{1}\right)\right|,\left|\sigma_{c}\left(p_{0}, p_{1}\right)\right|\right\} .
\end{aligned}
$$

Therefore

$$
\lim _{n \rightarrow \infty} \max \left\{\left|\sigma_{c}\left(p_{n}, p_{n+1}\right)\right|,\left|\sigma_{c}\left(q_{n}, q_{n+1}\right)\right|\right\}=0,
$$

which in turn gives

$$
\lim _{n \rightarrow \infty}\left|\sigma_{c}\left(p_{n}, p_{n+1}\right)\right|=0 \text { and } \lim _{n \rightarrow \infty}\left|\sigma_{c}\left(q_{n}, q_{n+1}\right)\right|=0 .
$$

For each $n>m$, we have

$$
\begin{aligned}
\sigma_{c}\left(p_{n}, p_{m}\right) & \preceq \sigma_{c}\left(p_{n}, p_{n-1}\right)+\sigma_{c}\left(p_{n-1}, p_{n-2}\right)-\sigma_{c}\left(p_{n-1}, p_{n-1}\right) \\
& +\sigma_{c}\left(p_{n-2}, p_{n-3}\right)+\sigma_{c}\left(p_{n-3}, p_{n-4}\right)-\sigma_{c}\left(p_{n-3}, p_{n-3}\right) \\
& +\cdots+\sigma_{c}\left(p_{m+2}, p_{m+1}\right)+\sigma_{c}\left(p_{m+1}, p_{m}\right)-\sigma_{c}\left(p_{m+1}, p_{m+1}\right) \\
& \preceq \sigma_{c}\left(p_{n}, p_{n-1}\right)+\sigma_{c}\left(p_{n-1}, p_{n-2}\right)+\cdots+\sigma_{c}\left(p_{m+1}, p_{m}\right),
\end{aligned}
$$

which implies that

$$
\left|\sigma_{\mathfrak{c}}\left(p_{n}, p_{m}\right)\right| \leqslant\left|\sigma_{\mathcal{c}}\left(p_{n}, p_{n-1}\right)\right|+\left|\sigma_{\mathfrak{c}}\left(p_{n-1}, p_{n-2}\right)\right|+\cdots+\left|\sigma_{\mathfrak{c}}\left(p_{m+1}, p_{m}\right)\right|
$$


As $n, m \rightarrow \infty$, we obtain

$$
\lim _{n, m \rightarrow \infty}\left|\sigma_{c}\left(p_{n}, p_{m}\right)\right|=0
$$

Similarly, one can prove that

$$
\lim _{n \rightarrow \infty}\left|\sigma_{c}\left(q_{n}, q_{m}\right)\right|=0,
$$

which implies that $\left\{p_{n}\right\}$ and $\left\{q_{n}\right\}$ are Cauchy sequences in $\left(Y, \sigma_{c}\right)$. Since, complex partial metric space $\left(\mathrm{Y}, \sigma_{\mathrm{c}}\right)$ is complete, there exist $\mathrm{p}, \mathrm{q} \in \mathrm{Y}$ such that $\left\{\mathrm{p}_{\mathrm{n}}\right\} \rightarrow \mathrm{p}$ and $\mathrm{q}_{\mathrm{n}} \rightarrow \mathrm{q}$ as $\mathrm{n} \rightarrow \infty$ and $\sigma_{\mathrm{c}}(\mathrm{p}, \mathrm{p})=$ $\lim _{n \rightarrow \infty} \sigma_{c}\left(p, p_{n}\right)=\lim _{n, m \rightarrow \infty} \sigma_{c}\left(p_{n}, p_{m}\right)=0, \sigma_{c}(q, q)=\lim _{n \rightarrow \infty} \sigma_{c}\left(q, q_{n}\right)=\lim _{n, m \rightarrow \infty} \sigma_{c}\left(q_{n}, q_{m}\right)=$ 0 . We now show that $p=\psi(p, q)$. Now,

$$
\begin{aligned}
\sigma_{c}(p, \psi(p, q)) & \preceq \sigma_{c}\left(p, p_{n+1}\right)+\sigma_{c}\left(p_{n+1}, \psi(p, q)\right) \\
& =\sigma_{c}\left(p, p_{n+1}\right)+\sigma_{c}\left(\psi\left(p_{n}, q_{n}\right), \psi(p, q)\right) \\
& \preceq \sigma_{c}\left(p, p_{n+1}\right)+r \max \left\{\sigma_{c}\left(p_{n}, p\right), \sigma_{c}\left(q_{n}, q\right), \sigma_{c}\left(\psi\left(p_{n}, q_{n}\right), p_{n}\right), \sigma_{c}(\psi(p, q), p)\right\} \\
& =\sigma_{c}\left(p, p_{n+1}\right)+r \max \left\{\sigma_{c}\left(p_{n}, p\right), \sigma_{c}\left(q_{n}, q\right), \sigma_{c}\left(p_{n+1}, p_{n}\right), \sigma_{c}(\psi(p, q), p)\right\},
\end{aligned}
$$

which implies that

$$
\left|\sigma_{c}(p, \psi(p, q))\right| \leqslant\left|\sigma_{c}\left(p, p_{n+1}\right)\right|+r \max \left\{\left|\sigma_{c}\left(p_{n}, p\right)\right|,\left|\sigma_{c}\left(q_{n}, q\right)\right|,\left|\sigma_{c}\left(p_{n+1}, p_{n}\right)\right|,\left|\sigma_{c}(\psi(p, q), p)\right|\right\} .
$$

As $n \rightarrow \infty,\left|\sigma_{\mathcal{c}}(p, \psi(p, q))\right| \leqslant r\left|\sigma_{\mathfrak{c}}(\psi(p, q), p)\right|$. Since $r \in[0,1)$, therefore $\left|\sigma_{\mathfrak{c}}(p, \psi(p, q))\right|=0 \Longrightarrow p=$ $\psi(p, q)$. Similarly we can prove that $q=\psi(q, p)$. Thus $(p, q)$ is a coupled fixed point of $\psi$. Now, if $(g, h)$ is another coupled fixed point of $\psi$, then

$$
\begin{aligned}
\sigma_{\mathcal{c}}(p, g)=\sigma_{\mathfrak{c}}(\psi(p, q), \psi(g, h)) & \preceq \max \left\{\sigma_{\mathfrak{c}}(p, g), \sigma_{\mathfrak{c}}(q, h), \sigma_{\mathfrak{c}}(\psi(p, q), p), \sigma_{\mathcal{c}}(\psi(g, h), g)\right\} \\
& \preceq \operatorname{rmax}\left\{\sigma_{\mathfrak{c}}(p, g), \sigma_{\mathfrak{c}}(q, h), \sigma_{\mathfrak{c}}(p, p), \sigma_{\mathfrak{c}}(g, g)\right\} .
\end{aligned}
$$

Since $\sigma_{\mathfrak{c}}(p, p) \preceq \sigma_{\mathcal{c}}(p, g)$ and $\sigma_{\mathcal{c}}(g, g) \preceq \sigma_{\mathcal{c}}(p, g)$, we have

$$
\sigma_{\mathcal{c}}(p, g) \preceq r \max \left\{\sigma_{\mathcal{c}}(p, g), \sigma_{\mathcal{c}}(q, h)\right\}, \quad\left|\sigma_{\mathcal{c}}(p, g)\right| \leqslant r \max \left\{\left|\sigma_{\mathcal{c}}(p, g)\right|,\left|\sigma_{\mathcal{c}}(q, h)\right|\right\} .
$$

Similarly, we can prove

$$
\left|\sigma_{\mathrm{c}}(\mathrm{q}, \mathrm{h})\right| \leqslant \operatorname{rmax}\left\{\left|\sigma_{\mathrm{c}}(\mathrm{p}, \mathrm{g})\right|,\left|\sigma_{\mathrm{c}}(\mathrm{q}, \mathrm{h})\right|\right\} .
$$

From (3.16) and (3.17), we have

$$
\max \left\{\left|\sigma_{\mathbf{c}}(p, g)\right|,\left|\sigma_{\mathcal{c}}(q, h)\right|\right\} \leqslant \max \left\{\left|\sigma_{c}(p, g)\right|,\left|\sigma_{c}(q, h)\right|\right\} .
$$

Since $0 \leqslant r<1$, we have $\max \left\{\left|\sigma_{\mathcal{c}}(p, g)\right|,\left|\sigma_{\mathcal{c}}(q, h)\right|\right\}=0$ implies that $\sigma_{\mathfrak{c}}(p, g)=0$ and $\sigma_{\mathcal{c}}(q, h)=0$. Therefore, $p=g$ and $q=h \Longrightarrow(p, q)=(g, h)$. Thus, $\psi$ has a unique coupled fixed point.

Corollary 3.7. Let $\left(\mathrm{Y}, \sigma_{\mathrm{c}}\right)$ be a complete CPMS. Suppose that the mapping $\psi: \mathrm{Y} \times \mathrm{Y} \rightarrow \mathrm{Y}$ satisfies

$$
\sigma_{\mathcal{c}}(\psi(p, q), \psi(r, s)) \preceq a \sigma_{c}(p, r)+b \sigma_{c}(q, s)+c \sigma_{c}(\psi(p, q), p)+d \sigma_{c}(\psi(r, s), r),
$$

for all $\mathrm{p}, \mathrm{q}, \mathrm{r}, \mathrm{s} \in \mathrm{Y}$, where $\mathrm{a}, \mathrm{b}, \mathrm{c}$ and $\mathrm{d}$ are non-negative constants with $\mathrm{a}+\mathrm{b}+\mathrm{c}+\mathrm{d}<1$, then $\psi$ has a unique coupled fixed point.

Proof. The proof follows from Theorem 3.6. Note that

$$
\begin{aligned}
& a \sigma_{c}(p, r)+b \sigma_{c}(q, s)+c \sigma_{c}(\psi(p, q), p)+d \sigma_{c}(\psi(r, s), r) \\
& \quad \leqslant r \max \left\{\sigma_{c}(p, r), \sigma_{c}(q, s), \sigma_{c}(\psi(p, q), p), \sigma_{c}(\psi(r, s), r)\right\},
\end{aligned}
$$

where $r=a+b+c+d<1$. 
Example 3.8. Let $Y=[0, \infty)$ endowed with the usual complex partial metric $\sigma_{c}: Y \times Y \rightarrow[0, \infty)$ defined by $\sigma_{c}(p, q)=\max \{p, q\}(1+i)$. The complex partial metric space $\left(Y, \sigma_{c}\right)$ is complete because $\left(Y, \sigma_{c}^{t}\right)$ is complete. Indeed, for any $p, q \in Y$,

$$
\sigma_{c}^{t}=2 \sigma_{c}(p, r)-\sigma_{c}(p, p)-\sigma_{c}(r, r)=2 \max \{p, q\}(1+i)-(p+i p)-(q+i q)=|p-q|+i|p-q| .
$$

Thus, $\left(\mathrm{Y}, \sigma_{\mathrm{c}}\right)$ is the Euclidean complex metric space which is complete. Consider the mapping $\psi: \mathrm{Y} \times \mathrm{Y} \rightarrow$ $\mathrm{Y}$ defined by $\psi(p, q)=\frac{|p-q|}{2}$. For any $p, q, g, h \in Y$, we have

$$
\begin{aligned}
\sigma_{\mathcal{c}}(\psi(p, q), \psi(g, h)) & =\frac{1}{2} \max \{|p-q|,|g-h|\}(1+i) \\
& =\frac{1}{2} \max \{p-q, q-p, g-h, h-g\}(1+i) \\
& \preceq \frac{1}{2} \max \{p, q, g, h\}(1+i) \\
& =\frac{1}{2} \max \left\{\sigma_{\mathfrak{c}}(p, g), \sigma_{\mathcal{c}}(q, h)\right\} \\
& \preceq \frac{1}{2} \max \left\{\sigma_{\mathfrak{c}}(p, g), \sigma_{\mathfrak{c}}(q, h), \sigma_{\mathfrak{c}}(\psi(p, q), p), \sigma_{\mathfrak{c}}(\psi(g, h), g)\right\}
\end{aligned}
$$

Thus, $\psi$ has a unique coupled fixed point. Here, $(0,0)$ is the unique fixed point of $\psi$.

\section{References}

[1] I. Altun, F. Sola, H. Simsek, Generalized contractions on partial metric spaces, Topology Appl., 157 (2010), $2778-2785$. 1

[2] H. Aydi, Some Coupled fixed point results on partial metric spaces, Int. J. Math. Math. Sci., 2011 (2011), 11 pages. 1, 2

[3] A. Azam, B. Fisher, M. Khan Common fixed point theorems in complex valued metric spaces, Numer. Funct. Anal. Optim., 32 (2011), 243-253. 1, 2.1

[4] T. G. Bhaskar, V. Lakshmikantham, Fixed point theorems in partially ordered metric spaces and applications, Nonlinear Anal., 65 (2006), 1379-1393. 1

[5] P. Dhivya, M. Marudai, Common fixed point theorems for mappings satisfying a contractive condition of rational expression on a ordered complex partial metric space, Cogent Math., 4 (2017), 10 pages 1, 2.6, 2.7, 2.10, 2.11, 2.12, 2.13

[6] E. Karapinar, K. Taş, V. Rakočević, Advances on Fixed Point Results on Partial Metric Spaces, Springer, Cham, (2019). 1

[7] V. Lakshmikantham, L. Ćirić, Coupled fixed point theorems for nonlinear contractions in partially ordered metric spaces and applications, Nonlinear Anal., 70 (2009), 4341-4349. 1

[8] S. G. Matthews, Partial Metric Spaces, In: $8^{\text {th }}$ British Colloquium for Theoretical Computer Science, Research Report 212, Dept. of Computer Science, University of Warwick, (1992). 1, 2.3, 2.4

[9] S. G. Matthews, Partial metric topology, Proc. $8^{\text {th }}$ summer conference on General topology and Applications, New York Acad. Sci., New York, 728 (1994), 183-197. 1, 2.4

[10] K. P. R. Rao, A. Sombabu, Suzuki type common fixed point theorems for four maps using $\alpha$-admissible in partial ordered complex partial metric spaces, Commun. Nonlinear Anal., 5 (2018), 40-54. 2.14

[11] F. Sabetghadam, H. P. Masiha, A. H. Sanatpour, Some coupled fixed point theorems in cone metric spaces, Fixed Point Theory Appl., 2009 (2009), 8 pages. 1

[12] B. Samet, C. Vetro, F. Vetro, From metric spaces to partial metric spaces, Fixed Point Theory Appl., 2013 (2013), 11 pages.

[13] W. Shatanawi, H. K. Nashine, N. Talat, Generalization of some coupled fixed point results on partial metric spaces, Int. J. Math. Math. Sci., 2012 (2012), 10 pages. 1, 2 